\title{
Association between smoking and cognitive impairment in multiple sclerosis
}

\author{
This article was published in the following Dove Press journal: \\ Neuropsychiatric Disease and Treatment \\ 10 September 2014 \\ Number of times this article has been viewed
}

\author{
Muhammed Emin Özcan' \\ Bahri İnce ${ }^{2}$ \\ Ayhan Bingöl ${ }^{3}$ \\ Simge Ertürk ${ }^{3}$ \\ Meriç Adil Altınöz ${ }^{4}$ \\ Hasan Hüseyin Karadeli' \\ Abdulkadir Koçer ${ }^{5}$ \\ Talip Asil' \\ 'Deparment of Neurology, \\ Medical Faculty, Bezmialem Vakıf \\ University, Istanbul, Turkey; \\ ${ }^{2}$ Department of Psychiatry, Bakırköy \\ Research and Training Hospital for \\ Psychiatry, Istanbul, Turkey; ${ }^{3}$ Mayıs \\ Psychology Center, Istanbul, Turkey; \\ ${ }^{4}$ Department of Molecular Biology \\ and Genetics, Haliç University, \\ Istanbul, Turkey; ${ }^{5}$ Department \\ of Neurology, Medical Faculty, \\ istanbul Medeniyet University, \\ Göztepe, Istanbul, Turkey
}

Correspondence: Muhammed Emin

Özcan

Department of Neurology, Bezmialem

Vakif University, Medical Faculty, Adnan

Menderes Bulvarı 34093 Fatih, İstanbul,

Turkey

Tel +90 2I2453 I700

Email emozcan@gmail.com
Purpose: Although smoking is known to cause various symptoms in multiple sclerosis (MS) patients, there have been no reports regarding the relationship between smoking and cognitive impairment in MS. Studying the effects of cigarette smoking in MS patients is imperative as there is a high prevalence of cognitive impairment in MS patients. In this study we examined the potentially deleterious effects of heavy smoking on mentation of patients with MS.

Patients and methods: MS patients receiving care at the Neurology Clinic at Bezmialem Vakıf University, between the ages of 18-65 years who have at least graduated elementary school were included in the study. The Brief Repeatable Battery of Neuropsychological Tests (BRB-N) is a commonly used method to assess cognitive function in MS patients and was utilized in our study. Patients that smoked for at least 10 pack-years were considered heavy smokers.

Results: All the patients were stratified into two groups: heavy smokers $(n=20)$ and nonsmokers $(n=24)$. For heavy smokers, their cognitive functioning was more impaired than that of nonsmokers $\left(P=0.04, \chi^{2}=4.227\right)$. For patients with cognitive impairment, $78.9 \%$ of the Paced Auditory Serial Addition Test and $63.2 \%$ of the Symbol Digit Modalities Test scores were found to be lower.

Conclusion: Previous reports have suggested that smoking increases the frequency of relapse among individuals with relapsing-remitting MS and accelerates disease progression in patients with progressive MS. According to the results of our study, heavy smokers had increased cognitive impairment when compared to nonsmokers. Extensive studies are necessary to further elucidate the relationship between smoking and cognitive impairment in MS patients.

Keywords: cigarette, BRB-N, nicotine, cognition, tobacco, mentation, memory

\section{Introduction}

Multiple sclerosis (MS) is an inflammatory disease characterized by damage to the myelin sheath surrounding neurons in the central nervous system (CNS). This damage may be progressive, or it may wax and wane with periods of relapse and remission. Motor, somatosensory, visual, cognitive, and psychiatric deficits may occur in MS patients depending on the location and size of the focal demyelination in the CNS. Cognitive impairments may manifest in the early phases of the clinical course of MS. ${ }^{1}$ In fact, the prevalence of cognitive deficits in MS patients ranges from $43 \%-70 \%{ }^{2}$

Cognitive impairments may prevent patients from being able to perform activities of daily living. Studies have shown that patients with cognitive deficits tend to avoid social activities, have higher rates of unemployment, and have difficulties in performing routine household chores. As such, the quality of life for these patients becomes further compromised as the degree of cognitive impairment increases. ${ }^{3}$ Due to the limited treatment options for $\mathrm{MS},{ }^{4}$ it is critical to understand what agents may worsen clinical symptoms. 
Cigarette smoking may trigger MS, ${ }^{5}$ facilitate the transformation of a clinically isolated syndrome into MS, ${ }^{6}$ increase MS relapse frequency, ${ }^{7}$ and promote progression of the disease. ${ }^{8}$ However, smoking is also known to increase cognitive performance via its effects on nicotinic acetylcholine receptors. ${ }^{9,10}$ Even though it is understood that smoking affects the clinical course of MS, there are no studies that have investigated the relationship between smoking and cognitive impairment in MS patients. On the other hand, there are many studies evaluating MS patients, and the relationship between cannabis use and cognitive impairment. ${ }^{11,12}$ Cigarette smoking exerts both positive and negative effects on cognition, and due to the high prevalence of cognitive impairment in MS patients, it is imperative to elucidate the effects of smoking on cognition in these patients. In this study we examined the potentially deleterious effects of heavy smoking on mentation in patients with MS.

\section{Material and methods}

MS patients that presented to the Neurology Clinic at Bezmialem Vakıf University (Istanbul, Turkey) were enrolled in the study once informed consent was obtained. A total of 72 consecutive patients presenting to the clinic between January 2013 and November 2013 met the inclusion criteria for this study and voluntarily agreed to participate it. Inclusion criteria were that the patients meet the Revised McDonald's Diagnostic Criteria (2010) for a diagnosis of MS, ${ }^{13}$ fall between 18-65 years old, and have at least graduated elementary school. Patients with cognitive impairment due to a condition other than MS; with a known psychiatric disorder; taking medications with cognitive side effects including anticholinergics and benzodiazepines; or who had received glucocorticoid treatments within the last 30 days were excluded from the study. Subjective symptomatic depression was evaluated with the Beck Depression Inventory.

\section{Demographics and MS evaluation}

Each patient was assessed by a neurologist with expertise in treating MS. Demographics including age, sex, and education were recorded. Information on the type of MS, clinical course, and duration of disease were obtained from reviewing patient charts. The extent of physical disability was measured using the Expanded Disability Status Scale (EDSS).

\section{Smoking history}

Patients were asked about their smoking habits and the frequency of smoking. Smoking rate was calculated with the following formula: (number of cigarettes per day $\times$ years of smoking)/20. Patients that were currently smoking and smoked for at least 10 pack-years were considered heavy smokers. ${ }^{14}$

\section{Evaluating cognition}

The Brief Repeatable Battery of Neuropsychological Tests (BRB-N) is a commonly utilized method for evaluating cognitive impairment in MS patients, with high sensitivity and specificity. ${ }^{15}$ The BRB-N was administered by an experienced neuropsychologist, and the presence of cognitive impairment and the specific areas of debility were detected with this test. The subtests of the BRB-N test include the Selective Reminding Test (SRT) and SRT-delayed recall (SRT-DR), Spatial Recall Test (SPART) and SPARTdelayed recall (SPART-DR), Symbol Digit Modalities Test (SDMT), Paced Auditory Serial Addition Test (PASAT), and Word List Generation (WLG). Patients scoring in the fifth percentile or less in at least two of these tests were considered to have cognitive impairment according to the normative data from the general Turkish population. ${ }^{16}$

\section{Statistical analysis}

All statistical analyses were completed using SPSS version 20 (IBM Corp., Armonk, NY, USA). Data were compared to a normal distribution using the Kolmogorov-Smirnov test and histograms. Data that were normally distributed were analyzed with the Student's $t$-test, and data that were not normally distributed were analyzed with the Mann-Whitney $U$-test. Ratios were compared with the $\chi^{2}$ test. Backward stepwise logistic regression was used to identify the factors that were statistically predictive of cognitive impairment. A $P$-value of $<0.05$ was considered statistically significant.

\section{Ethics board approval}

The study protocol was reviewed and approved by the Istanbul Bezmialem Vakıf University Ethics Committee. Informed consent documentation was collected from each patient after the research study was explained in full detail.

\section{Results}

\section{Patient sample}

A total of 72 patients were enrolled in the study, their average age (mean \pm standard deviation) was $36.54 \pm 10.29$ years, and $36.1 \%$ were males. Out of all patients, $80.6 \%$ had relapsing-remitting MS, $13.9 \%$ had secondary progressive MS, and 5.6\% had primary progressive MS. The mean duration of having an MS diagnosis was $6.57 \pm 6.13$ years. On average, the patients received $8.74 \pm 3.68$ years of schooling. The mean EDSS value 
Table I Demographics and clinical characteristics

\begin{tabular}{|c|c|c|c|c|}
\hline & Nonsmokers $(n=24)$ & Heavy smokers $(n=20)$ & $t, z$, or $\chi^{2}$ & P-value \\
\hline Age, years & $34.92 \pm 12.5$ & $40.10 \pm 7.25$ & $t=1.715$ & 0.095 \\
\hline Sex, male:female & $9: 15$ & $13: 7$ & $\chi^{2}=3.30$ & 0.069 \\
\hline Education duration, years & $8.83 \pm 3.65$ & $8.50 \pm 3.77$ & $z=0.273$ & 0.785 \\
\hline Duration of MS diagnosis, years & $5.88 \pm 6.35$ & $6.40 \pm 5.88$ & $z=0.42$ & 0.675 \\
\hline EDSS score, average (range) & $\mathrm{I} .75(\mathrm{I} .0-7.0)$ & $1.5(1.0-6.5)$ & $z=0.17$ & 0.865 \\
\hline Beck Depression Inventory score & $16.04 \pm 13.70$ & $17.05 \pm 1 \mid .57$ & $z=0.65$ & 0.516 \\
\hline MS types & & & $\chi^{2}=1.38$ & 0.5 \\
\hline - Relapsing-remitting, $\mathrm{n}$ & 18 & 16 & & \\
\hline - Secondary progressive, $n$ & 5 & 2 & & \\
\hline - Primary progressive, $n$ & I & 2 & & \\
\hline
\end{tabular}

Note: Data are expressed as the mean \pm standard deviation unless otherwise specified. Abbreviations: MS, multiple sclerosis; EDSS, Expanded Disability Status Scale.

was 1.5 and scores ranged from 1-7.5. It was determined that $41.7 \%$ were smokers, and of these patients, $53.3 \%$ were male. Out of the initial group of 72 patients, 28 patients did not meet the criteria for heavy cigarette smoking and therefore, were not included in the statistical analysis. Eighteen of the excluded patients were former smokers, while ten patients were excluded due as a result of tobacco consumption of less than 10 pack-years. Among the patients that were included in the study, the heavy smoking group consisted of 20 patients and the nonsmoker group included 24 patients.

\section{Comparing heavy smokers with nonsmokers}

Demographics and MS characteristics are summarized for heavy smokers $(n=20)$ and nonsmokers $(n=24)$ in Table 1. The cognitive scores for nonsmokers and heavy smokers are compared in Table 2. Cognitive impairment was identified in seven out of 24 nonsmokers $(29.2 \%)$ and in 12 out of 20 heavy smokers $(60 \%)$. Despite the lack of statistically significant differences between these groups in terms of subtest scoring, cognitive impairment was significantly greater $(P=0.04)$ in heavy smokers. In patients with cognitive impairment, the PASAT (78.9\%) and SDMT (63.2\%) test results were shown to be the most abnormal.

A logistic regression model was used to evaluate age, sex, education level, disease duration, EDSS score, and smoking status as independent predictors of cognitive impairment. The results demonstrate that EDSS and smoking status are independently associated with cognitive impairment in patients with MS (Table 3).

\section{Discussion}

Previous studies have identified both the positive ${ }^{17,18}$ and negative effects ${ }^{19,20}$ of smoking on the cognitive function. We demonstrated that cognitive impairment is significantly higher in MS patients that are heavy smokers. Our data are supported by other studies that have shown that smoking worsens MS symptoms. ${ }^{21,22}$ However, this study is the first to report the effects of smoking on cognition in MS patients.

In the $72 \mathrm{MS}$ patients that participated in this study, it was found that $41.7 \%$ smoked, which is higher than the general Turkish population at $27 \%$ according to the Turkish Statistical Institute (TurkStat). ${ }^{23}$ Our results contradict those of Friend et $\mathrm{al}^{14}$ as they reported lower smoking rates in the

Table 2 Cognitive score comparisons between heavy smokers and nonsmokers

\begin{tabular}{|c|c|c|c|c|}
\hline & Nonsmokers $(n=24)$ & Heavy smoker $(n=20)$ & $t, z$, or $\chi^{2}$ & $P$-value \\
\hline PASAT & $40.04 \pm 35.27$ & $33.40 \pm 32.80$ & $z=0.562$ & 0.574 \\
\hline SRT & $45.17 \pm 10.32$ & $45.25 \pm 8.38$ & $t=0.029$ & 0.977 \\
\hline SRT-DR & $7.33 \pm 2.58$ & $6.60 \pm 3.36$ & $t=0.818$ & 0.418 \\
\hline SPART & $16.13 \pm 5.15$ & $|4.50 \pm 6.7|$ & $t=0.909$ & 0.369 \\
\hline SPART-DR & $5.25 \pm 1.75$ & $4.75 \pm 2.34$ & $t=0.81 \mathrm{I}$ & 0.422 \\
\hline SDMT & $31.58 \pm 15.87$ & $28.95 \pm 10.54$ & $z=0.602$ & 0.547 \\
\hline WLG & $17.92 \pm 3.74$ & $19.05 \pm 5.39$ & $t=0.820$ & 0.417 \\
\hline Cognitive impairment (\%) & 29.2 & 60.0 & $\chi^{2}=4.227$ & 0.04 \\
\hline
\end{tabular}

Notes: Data are expressed as the mean \pm standard deviation unless otherwise specified. Patients scoring in the fifth percentile or less in at least two of these tests were considered to have cognitive impairment according to the normative data from the general Turkish population. Bold indicates a statistically significant $P$-value.

Abbreviations: PASAT, Paced Auditory Serial Addition Test; SRT, Selective Reminding Test; SRT-DR, SRT-delayed recall; SPART, Spatial Recall Test; SPART-DR, SPART-delayed recall; SDMT, Symbol Digit Modalities Test; WLG, word list generation. 
Table 3 Results of the logistic regression model for predictors of cognitive impairment

\begin{tabular}{lllll}
\hline & $\beta(\mathrm{SE})$ & $\boldsymbol{P}$-value & \multicolumn{2}{c}{$\mathbf{9 5 \% \text { Cl for odds ratio }}$} \\
\cline { 5 - 5 } & & Lower & Odds ratio \\
\hline Constant & $-1.48(0.62)$ & 1.091 & 1.594 \\
EDSS & $0.47(0.19)$ & 0.016 & 1.124 & 2.327 \\
Smoking status & $0.84(0.37)$ & 0.023 & & 4.816 \\
\hline (heavy smoking) & & & & \\
\hline
\end{tabular}

Notes: $R^{2}=0.19$ (Hosmer-Lemeshow test), 0.23 (Cox and Snell), 0.30 (Nagelkerke). Model $\chi^{2}(2)=11.265, P=0.004$.

Abbreviations: $\mathrm{Cl}$, confidence interval; EDSS, Expanded Disability Status Scale; SE, standard error.

sample of MS patients that they studied. The high number of smokers in our study may be due to the small patient sample size and insufficient public health education regarding smoking in that area of the country.

The prevalence of cognitive impairment ranges between $43 \%-70 \%$ in MS patients according to a previous study, and these deficits in cognition impact quality of life and employability. ${ }^{2}$ Memory is most often affected and the quantity of patients that experience memory and learning problems are between $40 \%-60 \% .{ }^{24}$ In our study, there were no statistically significant differences between the battery of cognitive subtests. Yet the testing demonstrated that the greatest deficits were in the PASAT and SDMT, which demonstrated cognitive impairments in $78.9 \%$ and $63.2 \%$ of the patients, respectively. These two tests are specifically utilized to assess working memory and how well individuals process information. Our cognitive testing results are consistent with previous literature..$^{25}$ The logistic regression analysis demonstrated that smoking status and EDSS scores are statistically significant predictors of cognitive impairment. There was no significant difference in EDSS scores between the smokers and nonsmokers.

There was a sex difference between heavy smokers and nonsmokers, but this discrepancy was not statistically significant. This difference might be due to fact that there is a higher proportion of male smokers as opposed to female smokers in the general population (approximately 3:1), and this is likely reflected in our MS patient sample. ${ }^{23}$ Additionally, previous studies have reported that male MS patients have greater cognitive impairments than females, ${ }^{26}$ but in a recent extensive study, it was found that sex is not associated with cognitive impairment. ${ }^{27}$

According to our results, cognitive impairment was found to be higher in heavy smokers as compared to nonsmokers among MS patients. The agonistic effects of nicotine on cholinergic receptors are thought to contribute to cholinergic dysfunction observed in patients with cognitive deficits. ${ }^{10}$ Moreover, the risk of developing cognitive impairments increases in smokers in cohort studies. In a meta-analysis of
19 prospective studies, Anstey et $\mathrm{al}^{28}$ reported that the risk of developing dementia in smokers was $70 \%$ higher than that of nonsmokers.

Even though it has been reported that nicotine has positive effects on cognition, the adverse effects of chronic smoking on cognition might be related to the high proportion of substances in the tar besides nicotine. Inflammatory changes induced by tar substances on cerebral artery endothelial surfaces in addition to the chronic exposure to carbon monoxide may lead to detrimental effects in CNS neurons over the long-term. In fact, in Scandinavian countries where moist snuff use is common, the risk of MS was higher in smokers as opposed to moist snuff users. Blood nicotine levels in moist snuff users are similar or higher in smokers, and their higher nicotine levels reportedly decrease MS risk. ${ }^{29,30}$ This suggests that cerebral tissue inflammation may arise from tar substances in cigarettes. If pure nicotine and cigarette smoke were compared in terms of their effects to cause cognitive impairments in an experimental setting, and nicotine is found to be beneficial for cognition, then nicotine may be considered for treating MS patients with cognitive impairments in the form of a patch or gum. Moreover, MS patients would be instructed to avoid smoking in order to reduce exposure to tar substances. There are significant limitations to the present study including the small sample size, the lack of a healthy control group, and reliance on patient self-reports concerning the details of tobacco consumption.

\section{Conclusion}

Our study demonstrated that heavy cigarette smoking negatively affected cognition in MS patients. In the future, we recommend that further studies are performed comparing cigarette smokers with moist snuff users in order to determine whether chronic nicotine exposure and/or cigarette tar substances contribute to cognitive impairment in MS patients. Nevertheless, smoking is a risk factor for the development of MS and cognitive deficits, and it is imperative that clinicians increase patient awareness regarding these risks. 


\section{Acknowledgment}

We would like to thank all volunteers for their participation in this study.

\section{Disclosure}

The authors report no conflicts of interest in this work.

\section{References}

1. Pelosi L, Geesken JM, Holly M, Hayward M, Blumhardt LD. Working memory impairment in early multiple sclerosis. Evidence from an eventrelated potential study of patients with clinically isolated myelopathy. Brain. 1997;120:2039-2058.

2. Rao S, Leo G, Bernardin L, Unverzagt F. Cognitive dysfunction in multiple sclerosis: frequency, patterns, and predictions. Neurology. 1991;41: 685-691.

3. Beatty WM, Blanco CR, Wilbanks SL, Paul RH, Hames KA. Demographic, clinical, and cognitive characteristics of multiple sclerosis patients who continue to work. J Neurol Rehab. 1995;9:167-173.

4. Pierson SH, Griffith N. Treatment of cognitive impairment in multiple sclerosis. Behav Neurol. 2006;17(1):53-67.

5. Jafari N, Hintzen RQ. The association between cigarette smoking and multiple sclerosis. J Neurol Sci. 2011;311(1-2):78-85.

6. Di Pauli F, Reindl M, Ehling R, et al. Smoking is a risk factor for early conversion to clinically definite multiple sclerosis. Mult Scler. 2008;14(8): 1026-1030.

7. D'hooghe MB, Nagels G, Bissay V, De Keyser J. Modifiable factors influencing relapses and disability in multiple sclerosis. Mult Scler. 2010; 16(7):773-785.

8. Pittas F, Ponsonby AL, van der Mei IA, et al. Smoking is associated with progressive disease course and increased progression in clinical disability in a prospective cohort of people with multiple sclerosis. J Neurol. 2009;256(4):577-585.

9. Wignall ND, de Wit H. Effects of nicotine on attention and inhibitory control in healthy nonsmokers. Exp Clin Psychopharmacol. 2011;19(3): 183-191.

10. Rusted JM, Sawyer R, Jones C, et al. Positive effects of nicotine on cognition: the deployment of attention for prospective memory. Psychopharmacology (Berl). 2009;202(1-3):93-102.

11. Ghaffar O, Feinstein A. Multiple sclerosis and cannabis: a cognitive and psychiatric study. Neurology. 2008;71(3):164-169.

12. Papathanasopoulos P, Messinis L, Lyros E, Kastellakis A, Panagis G. Multiple sclerosis, cannabinoids, and cognition. J Neuropsychiatry Clin Neurosci. 2008;20(1):36-51.

13. Polman CH, Reingold SC, Banwell B, et al. Diagnostic criteria for multiple sclerosis: 2010 revisions to the McDonald criteria. Ann Neurol. 2011;69(2):292-302.

14. Friend KB, Mernoff ST, Block P, Reeve G. Smoking rates and smoking cessation among individuals with multiple sclerosis. Disabil Rehabil. 2006;28(18):1135-1141.
15. Portaccio E, Goretti B, Zipoli V, et al. Reliability, practice effects and change indices for Rao's brief repeatable battery. Mult Scler. 2010;16: 611-617.

16. Bingöl A, Yıldız Ş, Topçular B, et al. Brief repeatable battery (BRB) Turkish normative data. Eur J Neurol. 2012;19(Suppl 1):458-807.

17. Caldirola D, Daccò S, Grassi M, et al. Effects of cigarette smoking on neuropsychological performance in mood disorders: a comparison between smoking and nonsmoking inpatients. J Clin Psychiatry. 2013;74(2):e130-e136.

18. Yoneda I, Watanabe Y. Comparisons of altitude tolerance and hypoxia symptoms between nonsmokers and habitual smokers. Aviat Space Environ Med. 1997;68(9):807-811.

19. Chamberlain SR, Odlaug BL, Schreiber LR, Grant JE. Association between tobacco smoking and cognitive functioning in young adults. Am J Addict. 2012;21(1):S14-S19.

20. Durazzo TC, Meyerhoff DJ, Nixon SJ. Chronic cigarette smoking: implications for neurocognition and brain neurobiology. Int J Environ Res Public Health. 2010;7(10):3760-3091.

21. Shirani A, Tremlett H. The effect of smoking on the symptoms and progression of multiple sclerosis: a review. J Inflamm Res. 2010;3: $115-126$.

22. Sundström P, Nyström L. Smoking worsens the prognosis in multiple sclerosis. Mult Scler. 2008;14(8):1031-1035.

23. Results of the Global Adult Tobacco Survey [webpage on the Internet]. Ankara, Turkey: Republic of Turkey Turkish Statistical Institute; 2012. Available from: http://www.turkstat.gov.tr/PreHaberBultenleri. do?id=13142. Accessed May 24, 2014.

24. Rao SM, Grafman J, DiGiulio D, et al. Memory dysfunction in multiple sclerosis: Its relation to working memory, semantic encoding, and implicit learning. Neuropsychology. 1993;7(3):364-374.

25. Brochet B, Deloire MS, Bonnet M, et al. Should SDMT substitute for PASAT in MSFC? A 5-year longitudinal study. Mult Scler. 2008;14(9): 1242-1249.

26. Savettieri G, Messina D, Andreoli V, et al. Gender-related effect of clinical and genetic variables on the cognitive impairment in multiple sclerosis. J Neurol. 2004;251(10):1208-1214.

27. Borghi M, Cavallo M, Carletto S, et al. Presence and significant determinants of cognitive impairment in a large sample of patients with multiple sclerosis. PLoS One. 2013;8(7):e69820.

28. Anstey KJ, von Sanden C, Salim A, O’Kearney R. Smoking as a risk factor for dementia and cognitive decline: a meta-analysis of prospective studies. Am J Epidemiol. 2007;166:367-378.

29. Hedström AK, Bäärnhielm M, Olsson T, Alfredsson L. Exposure to environmental tobacco smoke is associated with increased risk for multiple sclerosis. Mult Scler. 2011;17(7):788-793.

30. Hedström AK, Hillert J, Olsson T, Alfredsson L. Nicotine might have a protective effect in the etiology of multiple sclerosis. Mult Scler. 2013; 19(8):1009-1013.
Neuropsychiatric Disease and Treatment

\section{Publish your work in this journal}

Neuropsychiatric Disease and Treatment is an international, peerreviewed journal of clinical therapeutics and pharmacology focusing on concise rapid reporting of clinical or pre-clinical studies on a range of neuropsychiatric and neurological disorders. This journa is indexed on PubMed Central, the 'PsycINFO' database and CAS,

\section{Dovepress}

and is the official journal of The International Neuropsychiatric Association (INA). The manuscript management system is completely online and includes a very quick and fair peer-review system, which is all easy to use. Visit http://www.dovepress.com/testimonials.php to read real quotes from published authors. 